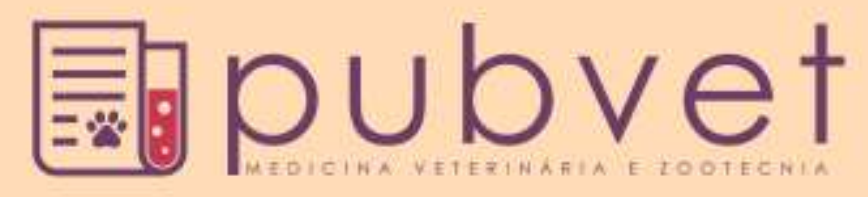

https://doi.org/10.22256/pubvet.v12n2a21.1-4

\title{
Hematoma aural em gato jovem: Relato de caso
}

\section{Raquel Albuquerque Silva ${ }^{6}$, Karoline Figueredo Rodrigues ${ }^{6} *$, Thays Garreto Rodrigues dos Santos ${ }^{\oplus 2}$, Thamara Barrozo Sampaio ${ }^{\oplus 1}$, Pablo Martins Veloso de Sousa 6 , Kellen Matuzzy Silva ${ }^{6}$, Thiago Sousa da Silva 1}

${ }^{1}$ Médico (a) Veterinária Residente em clínica e cirurgia de cães e gatos, Universidade Federal do Piauí, Teresina, Piauí, Brasil. ${ }^{2}$ Graduando em Medicina Veterinária, Universidade Federal do Piauí, Teresina, Piauí, Brasil.

${ }^{3}$ Médico (a) Veterinária Residente em anestesiologia veterinária, Universidade Federal do Piauí, Teresina, Piauí, Brasil.

*Autor para correspondência, E-mail: karol-figueredo@hotmail.com

RESUMO. Os otohematomas ou hematomas aurais são alterações resultantes de um acúmulo de sangue dentro da cartilagem auricular, ocasionados por diversos fatores, como otites de variadas etiologias. Existem vários procedimentos clínicos e cirúrgicos para o tratamento do otohematoma, porém nem todos trazem resultados satisfatórios. Este relato tem por objetivo relatar o caso de um felino, sem raça definida, de quatro anos e quatro meses de idade, diagnosticado com hematoma aural recidivante, devido à otite.

Palavras chave: Felino, hematoma aural, otite

\section{Aural hematoma in young cat: Case report}

\begin{abstract}
Otohematoma or aural hematoma are affections defined as an accumulation of blood within the atrial cartilage, caused by several factors, such as otitis of many etiologies. There are several clinical and surgical procedures for the treatment of otohematoma, but few with satisfactory results. The purpose of this arcticle is to report a case of a four year and four months old feline, diagnosed with recurrent aural hematoma due to otitis.
\end{abstract}

Keywords: Feline, aural hematoma, otitis

\section{Hematoma aural em gato jovem: Reporte de caso}

RESUMEN. Los otohematomas o hematomas aurales son alteraciones resultantes de una acumulación de sangre dentro del cartílago auricular, ocasionados por diversos factores, como otitis de variadas etiologías. Existen varios procedimientos clínicos y quirúrgicos para el tratamiento del otohematoma, pero no todos traen resultados satisfactorios. Este relato tiene por objetivo relatar el caso de un felino, mestizo, de cuatro años y cuatro meses de edad, diagnosticado con hematoma aural recidivante, debido a la otitis.

Palabras clave: Felino, hematoma aural, otitis

\section{Introdução}

O otohematoma ou hematoma aural são alterações comuns na clínica, sendo que em cães é considerado o oitavo procedimento mais realizado em cirurgias (Graça, 2010). Essa alteração é resultante de um acúmulo de sangue dentro da cartilagem auricular ocasionada por diversos fatores como otites de variadas etiologias (Rodrigues et al., 2016).
Na fase aguda do otohematoma observa-se uma deposição de fibrina oriunda de uma hemostasia fisiológica do organismo, com seroma sanguinolento. Na fase crônica, tem-se uma formação de fibrose compreendida em uma massa fixa na superfície côncava do pavilhão auricular, com consequente deformação (Evangelista et al., 2012). 
Existem vários procedimentos clínicos $\mathrm{e}$ cirúrgicos para o tratamento do otohematoma, porém nem todos trazem resultados satisfatórios, ocasionando problemas no tempo de recuperação, eficiência do método cirúrgico, além de perda estética (Schossler et al., 2007).

O tratamento clinico consiste na drenagem do sangue extravasado com auxílio de seringa hipodérmica e a lavagem da cavidade lesionada com solução fisiológica ou aplicação de corticosteroides. Já o tratamento cirúrgico consiste em incisão de pele associada ou não a suturas para abolição dos espaços adjacentes em forma de "S", longitudinais, paralelas, elípticas, circulares e a fixação da pele à cartilagem com pontos de Wolff (Eurides et al., 2008).

Portanto, este relato tem por objetivo relatar o caso de um felino, sem raça definida, de quatro anos e quatro meses de idade diagnosticada com hematoma aural recidivante devido à otite.

\section{Relato de Caso}

Foi atendido no Hospital Veterinário Universitário, Médico Veterinário Jeremias Pereira da Silva da Universidade Federal do Piauí, Teresina, Piauí, no dia 3 de março de 2017, um felino macho, sem raça definida, de quatro anos e quatro meses de idade, com $3,55 \mathrm{~kg}$. O animal apresentava um aumento de volume na orelha esquerda sendo que o mesmo já tinha sido submetido a um procedimento de drenagem com agulha hipodérmica (Figura 1). Tutor afirmou que animal tinha acesso à rua, coabitava com mais três felinos saudáveis, animal não era castrado, vermifugação atrasada assim como, vacinas antirrábica e de viroses desatualizadas.

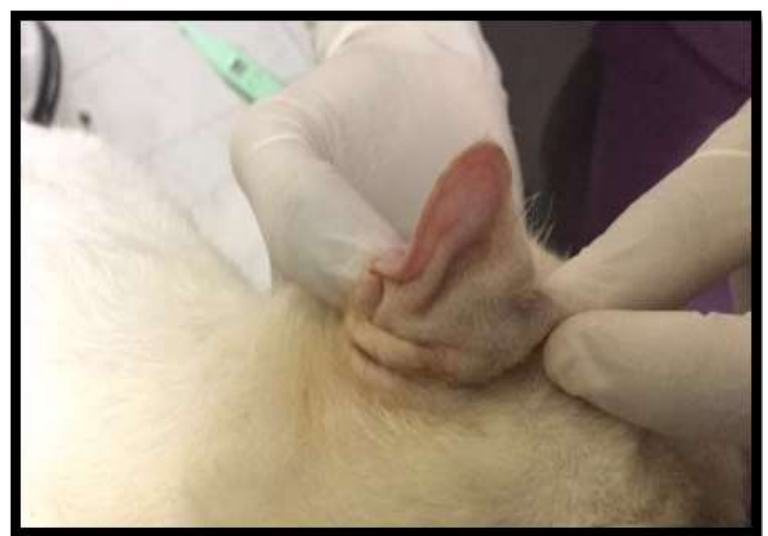

Figura 1. Aumento de volume na região auricular. Fonte: Arquivo pessoal.

Ao exame físico foi constatada mucosa ocular e oral normocoradas, linfonodos não reativos, presença de excesso de cerúmen em ambos os condutos auditivos, presença de aumento de volume na orelha esquerda o que era semelhante à otohematoma. Foi então prescrito medicações Auri Clean ${ }^{1}$ (limpeza do conduto auditivo) a cada 12 horas durante sete dias seguido de Natalene ${ }^{2}$ a cada 12 horas durante 7 dias, após o tratamento foi proposto uma nova reavaliação do animal.

No dia 10 de março de 2017 o animal retornou e segundo tutor, houve melhora no prurido otológico após uso das medicações prescritas. $\mathrm{O}$ animal se encontrava ativo, ingestão de água e alimento normal, fezes e urina normais. Ao exame físico foram constatadas mucosas ocular e oral normocoradas, tpc $<2 \mathrm{~s}$, animal hidratado, pelo brilhoso, sem alterações na ausculta cardiopulmonar assim como na palpação abdominal e linfonodos submandibiulares reativos.

Foram solicitados alguns exames laboratoriais como hemograma, bioquímica sérica (Ureia, Creatinina, Alanina Aminotransferase - ALT, Proteína Total, Albumina, Globulina e Fósforo), além de citologia de ouvido (direito e esquerdo).

Diante do quadro clínico do paciente suspeitou-se de otohematoma associado a otite do conduto auditivo. Os exames foram solicitados a fim de realizar o procedimento cirúrgico de drenagem do otohematoma no animal. Após resultados e análise dos exames laboratoriais, o animal foi encaminhado ao procedimento cirúrgico no dia 16 de março de 2017 (Figura 2) (Figura 3).

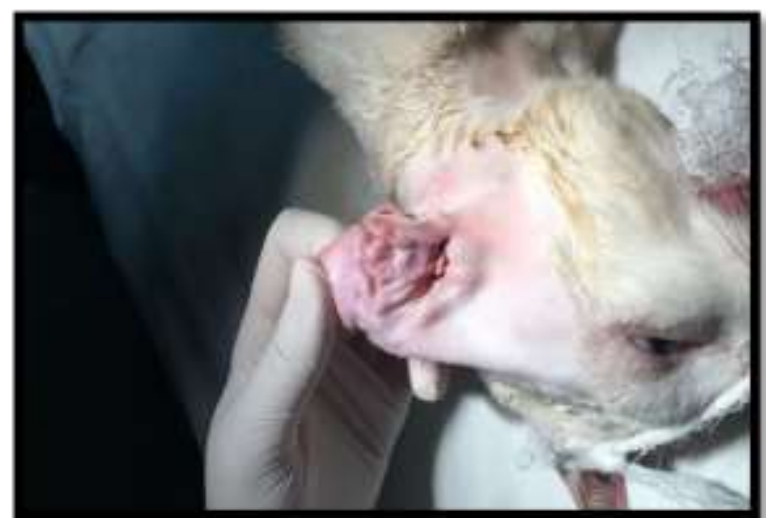

Figura 2. Aspecto do otohematoma pré- cirúrgico. Fonte: Arquivo pessoal.

${ }^{1}$ Produto para higiene do conduto auditivo contendo extrato de calêndula, extrato de aloe vera, propilenoglicol, PPG-5-Ceteth-20, essência de pêssego, fenoxietanol, metilparabeno, butilparabeno, etilparabeno, propilparabeno, PEG 75 lanolina, EDTA dissódico, glicerina, ácido cítrico, água.

${ }^{2}$ Antibiótico e antiinflamatório otológico a base de diazinon, pimaricina, neomicina e acetate de dexametasona. 


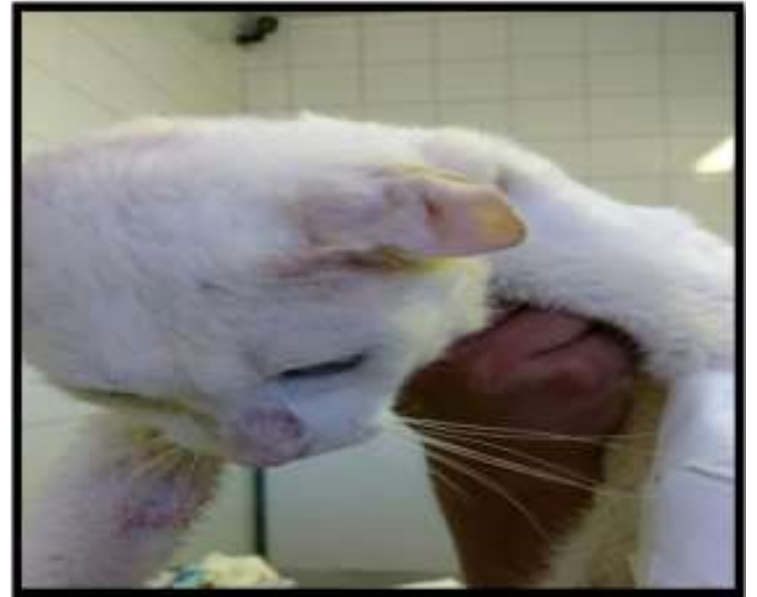

Figura 3. Aspecto do otohematoma pós-cirúrgico. Fonte: Arquivo pessoal.

\section{Discussão}

Suspeita-se de otohematoma com base no histórico, exame físico, exames complementares e achados citológicos sendo que o diagnóstico definitivo é obtido ao exame físico na qual se nota presença de tumefações flutuantes, tensas e as vezes dolorosas, que variam de tamanho e de posição (Santos, 2008), além disso, se associa ao histórico do animal como: choque da orelha com a cabeça do próprio animal, ato de coçar, inflamações, doenças que interferem nos fatores de coagulação, parasitas, alergias, e mesmo corpos estranhos (Schossler et al., 2007). Nesse relato o diagnóstico se deu em base ao exame clínico e ao histórico do animal.

Alguns autores relatam o intenso prurido presente na otite como fator relevante na formação do otohematoma (Birchard \& Sherding, 2008, Graça, 2010, Evangelista et al., 2012) por ser um sintoma que o animal na tentativa de amenizar, realiza movimentos bruscos com a cabeça ou até mesmo o ato de coçar intensa e constantemente, ocasionando na ruptura de vasos sanguíneos (Graça, 2010). Desta forma, pode-se associar o desenvolvimento do hematoma aural no felino então descrito à presença de otite e prurido.

$\mathrm{O}$ animal apresentou tumefações na orelha esquerda associada à presença de cerúmen em quantidade moderada em ambas as orelhas, o que de acordo com a literatura podem ser considerados alguns dos sinais e sintomas apresentados. Além disso, o paciente já havia sido submetido por procedimento de drenagem, porém a otite que o animal apresentava não havia sido tratada, o que segundo a literatura afirma que o otohematoma deve-se tratar, acima de tudo, a causa base da enfermidade.
A reincidência da patologia pode ser associada ao animal não estar na fase inicial da alteração, uma vez que Evangelista et al. (2012) descrevem que a aspiração de otohematomas na fase inicial utilizando agulha hipodérmica associado a lavagem da cavidade com solução fisiológica tendem a solucionar a alteração, em contrapartida realizar esse procedimento em casos de hematomas extensos se torna ineficiente e podem resultar em infecção secundária e retorno da alteração.

Schiochet et al. (2010) ainda confere a explicação da recidiva ao fato de que nem sempre o tratamento conservador de aspiração com agulha para drenagem é bem-sucedido, ainda mais quando realizado repetidas vezes. Tal afirmativa confere com o quadro relatado uma vez que o animal já havia passado por procedimento de aspiração do otohematoma porem obteve recidiva.

Dentre os fatores predisponentes à formação do otohematoma estão as otites interna, média ou externa. E essas podem ser decorrentes da conformação anatômica (Graça, 2010), prurido nas orelhas, corpos estranhos, tumores e pólipos no canal auditivo e ectoparasita (Graça, 2010).

Quanto aos parasitas que acometem os gatos, podemos citar o Otodectes cynotis, Sarcoptes scabiei, Notoedris cati, Cheyletiella spp. e Eutrombicula spp. Dentre esses, o primeiro tem maior ocorrência, sendo relatados em $50 \%$ dos casos de felinos com otite externa (Rosser, 2004), em contrapartida os demais são relatados em menos de 10\% dos casos (Graça, 2010).

As otites externas promovidas pela otocaríase, são resultantes da reação inflamatória provocada pela presença de apenas 2 ou 3 três ácaros. Vale frisar que o Octodectes cynotis adultos podem migrar para outras regiões do corpo do seu hospedeiro e ainda tem capacidade de sobreviver fora desse animal (Angus, 2004, Graça, 2010). Desta forma, a otite externa do presente paciente poderia ser ocasionada pela presença do parasita, contudo a não identificação destes nos exames do conduto auditivo podem estar associadas a pequenas cargas de ácaros ou a migração para outra parte do corpo (Angus, 2005, Graça, 2010). Com a cronicidade a otite externa pode ser acompanhada da produção de cerúmen e predispor o animal a infecções secundárias por leveduras ou bactérias (Rosser, 2004, Gotthelf, 2007, Graça, 2010). Semelhante ao que foi descrito e encontrado no exame citológico do paciente deste relato. Onde foi detectada a presença de 
quantidade moderada de leveduras semelhantes à Mallassezia sp e além disso, bactérias tipo cocos.

Segundo Tillmann et al. (2014), a otite pode ser uma das causas do otohematoma sendo que esta é caracterizada por uma inflamação do meato acústico podendo ser etiologia multifatorial e classificada em externa, média e interna dependendo da localização na orelha. Sendo que a otite externa parasitária é a mais comum em felino o que confere com o caso relatado.

Segundo a literatura, o otohematoma é mais frequentemente observado em cães, contudo ainda não se tem muitos relatos em felinos (Pacheco et al., 2013). Mas semelhante aos cães foi observado que o gato acometido pela patologia, manifestou otite simultaneamente, e sinais clínicos como pruridos intensos (Graça, 2010), cerúmen no conduto auditivo e aumento do pavilhão auricular (Evangelista et al., 2012). Prévio ao procedimento cirúrgico é necessária a investigação e a terapia corretiva da causa primária do otohematoma afim de evitar-se reincidências como afirmam Tillmann et al. (2014) corroborando com o que foi proposto no caso relatado.

\section{Conclusão}

O otohematoma é uma afecção clínico cirúrgica que raramente afeta felinos e que, pode estar associada à uma otite como causa primaria. Os exames laboratoriais como citologia associada ao exame clinico, e histórico do paciente são de suma importância para o fechamento do diagnóstico. No caso relatado, o tratamento inicial da causa primaria anterior a cirurgia foi crucial para que reduzir os riscos de recidiva do otohematoma uma vez que o mesmo já havia sido submetido por procedimento de drenagem com uso de agulha.

\section{Referências Bibliográficas}

Angus, J. C. 2004. Otic cytology in health and disease. Veterinary Clinics of North America: Small Animal Practice, 34, 411-424.

Angus, J. C. 2005. Pathogenesis of otitis externa: understanding primary causes. Proceeding of the North American Veterinary Conference.

Birchard, S. J. \& Sherding, R. G. 2008. Manual Saunders: clínica de pequenos animais, São Paulo.

Eurides, D., Souza, L. A., Oliveira, B. J. N. A. \& Luiz, A. F. S. 2008. Drenagem de otohematoma em cães. Revista Portuguesa de Ciências Veterinárias, 103, 59-63.

Evangelista, L. S. M., Carvalho, Y. N. T., Branco, M. A. C., Lopes, R. R. F. B., Neto, J. A. \& Quessada, A. M. 2012. Estudo retrospectivo do otohematoma em cães atendidos em um hospital veterinário. Acta Veterinaria Brasilica, 6, 48-51.

Gotthelf, L. N. 2007. Doenças do ouvido em pequenos animais. Editora Roca, São Paulo.

Graça, J. C. L. 2010. Otohematoma - estudo retrospectivo de 6 anos: possíveis etiologias. Departamento de Medicina Veterinária. Universidade Técnica de Lisboa, Lisboa.

Pacheco, A., Montanha, F., Gomes, D. \& Bernardi, C. 2013. Tratamento cirúrgico de otohematoma por colocação de brinco captonado em cão-Relato de caso. Revista Científica Eletrônica de Medicina Veterinária, 20, 1-8.

Rodrigues, N. M., Quessada, A. M., Silva, F. L., Silva, E., M.C., Costa Neto, J. M. \& Lima, W. C. 2016. Epidemiologia e risco anestésico de cães portadores de otohematoma. Acta Scientiae Veterinariae, 44, 1-6.

Rosser, E. J. 2004. Causes of otitis externa. Veterinary Clinics of North America: Small Animal Practice, 34, 459-468.

Santos, S. I. R. 2008. Otohematoma canino: Epidemiologia e Terapêutica. Dissertação de Mestrado em Medicina Veterinária. Lisboa.

Schiochet, F., Teixeira, E., Rodrigues, P. R. C., Gimosk, A. W., de Castro Beck, C. A., Contesini, E. A., Alievi, M. M. \& Fratini, L. M. 2010. Hematoma auricular em gato: relato de caso. Veterinária em Foco, 7, 198-205.

Schossler, J. E., Müller, D. \& Pinheiro, M. 2007. Proposição de técnica para drenagem de Otohematoma em cães. Arquivos de Ciências Veterinárias e Zoologia da UNIPAR, 10, 117119.

Tillmann, M. T., Mendes, C. B. D. M., Felix, A. d. O. C., Mueller, E. N. \& Oliveira Nobre, M. 2014. Fibrose auricular secundária a otohematoma em felino: terapêutica com glicocorticoide- relato de caso. Science And Animal Health, 2, 42-49.

Article History:

Received 17 September 2017

Accepted 11 October 2017

Available online 21 December 2017

License information: This is an open-access article distributed under the terms of the Creative Commons Attribution License 4.0, which permits unrestricted use, distribution, and reproduction in any medium, provided the original work is properly cited. 